[0212-7199 (2004) 21: 2; pp 53-55] ANALES DE MEDICINA INTERNA Copyright (C) 2004 ARAN EDICIONES, S.L. ..................................................... AN. MeD. InTERnA (Madrid) Vol. 21, N. $^{\circ} 2$, pp. 53-55, 2004

\title{
Oxigenoterapia continua domiciliaria: un buen tratamiento si se hace bien
}

Amilibia Alonso J, Sobradillo Peña V. Oxigenoterapia continua domiciliaria: un buen tratamiento si se hace bien. An Med Interna (Madrid) 2004; $21: 53-55$

La oxigenoterapia continua domiciliaria (OCD) es el tratamiento de la insuficiencia respiratoria crónica (IRC). La insuficiencia respiratoria es el resultado de una alteración en la ventilación alveolar, o bien en relación ventilación perfusión, o bien en ambas conjuntamente. Esta situación se alcanza en su forma crónica generalmente en pacientes con EPOC avanzado, pero también en otras patologías como la fibrosis pulmonar, neumoconiosis, alteraciones restrictivas de la caja torácica, trastornos neuromusculares, etc.

Los efectos beneficiosos de la OCD en la IRC han sido estudiados casi siempre en pacientes con EPOC y desde los últimos años $50(1,2)$ se describen disminución en las resistencias vasculares y en la hipertensión arterial pulmonar y mejoría en funciones neuropsíquicas (3,4). Son dos trabajos de 1980 y 1981 los que demuestran una mejoría en la supervivencia a largo plazo de los pacientes con oxígenoterapia y dan base a los actuales indicaciones de OCD: El americano NOTT (Nocturnal Oxygen Therapy Trial group) (3) y el británico MRC (British Medical Research Council) (5). Se admiten hoy en día las mismas indicaciones de OCD y los mismos beneficios en la IRC debida a otros procesos, no EPOC, aunque no existen evidencias demostradas.

Se han mostrado algunas realidades que obligan a hacer un esfuerzo continuo en la buena indicación y cumplimiento del tratamiento con OCD. En primer lugar es necesario que los pacientes realmente necesiten el tratamiento, esto es que cumplan las indicaciones de OCD: que presenten una $\mathrm{PO}_{2} \leq 55$ $\mathrm{mmHg}$ o bien $\mathrm{PO}_{2} 55-59 \mathrm{mmHg}$, si se acompaña de Cor Pulmonale, arritmias importantes o poliglobulia (Hcto>55), todo ello en situación basal (6). Esto es sin reagudizaciones en los últimos 3 meses y con un tratamiento farmacológico correcto. Para que se obtengan los beneficios esperados el flujo de oxígeno que se suministra debe ser capaz de revertir la hipoxemia, alcanzando una $\mathrm{PO}_{2} \geq 60$ o una $\mathrm{SaO}_{2} \geq 90 \%$ y el paciente debe utilizar el tratamiento al menos $15 \mathrm{~h} /$ día, incluyendo las horas de sueño. Es llamativo que estas condiciones se cumplen en menos del $25 \%$ de los casos en el estudio de Feu y cols. que se publica en éste número y también en estudios previos ya conocidos (7-9).

Por otro lado la OCD es un tratamiento frecuente habida cuenta la alta prevalencia de EPOC que el estudio IBERPOC demostró en 7 zonas geográficas diferentes $(10,11)$ es del $9 \%$ en personas de entre 40 y 69 años y llega al $15 \%$ en fumadores activos. Ese estudio demostró también que el $78 \%$ de las personas con EPOC no están diagnosticadas en nuestro medio y que solo el $49 \%$ de las EPOC graves reciben algún tratamiento. Se ha estimado previamente que el $15 \%$ de los pacientes diagnosticados de EPOC reciben OCD, en estudios realizados en el ámbito hospitalario (12). Así las cosas en la comunidad Autónoma del País Vasco actualmente existen 3100 tratamientos de OCD en una población de 2.000.000 de habitantes aproximadamente (150/100.000) lo que suponen 2.777.000 €/año, una cantidad que aunque probablemente solo representa escasamente el $4 \%$ del coste total del EPOC (IBERPOC) no deja de ser un esfuerzo económico importante que seguramente no se utiliza de forma óptima.

\section{CORRECTA UTILIZACIÓN DE OCD}

Hay acuerdo general en que se debe realizar una gasometría arterial en situación basal para indicar el tratamiento. Si el paciente cumple las condiciones gasométricas de la indicación de OCD se inicia el tratamiento asegurándose de que el flujo prescrito soluciona la hipoxemia, mediante una Pulsioximetría en la que se alcance una saturación de oxígeno $\left(\mathrm{SpO}_{2}\right)$ $\geq 92 \%$. En ese momento el paciente debe estar recibiendo un tratamiento farmacológico correcto.

El tratamiento correcto en EPOC debe incluir $\beta$ miméticos y anticolinérgicos inhalados, preferiblemente de acción prolongada y correctamente administrados. Esto no es fácil, ya que repetidamente se demuestra que en una alta proporción los pacientes no son capaces de realizar bien la maniobra de inhalación. La enseñanza correcta y la utilización de cámaras inhalatorias pueden mejorar el cumplimiento. Es aconsejable incluir corticoides inhalados ya que disminuyen las reagudizaciones y mejoran la calidad de vida (13). En los pacientes en los que se demuestre una mejoría en los parámetros espirométricos tras un tratamiento prolongado de corticoides inhalados, su administración se hace obligatoria (14). Las teofilinas tan utilizadas anteriormente presentan algunas dificultades por efectos secundarios y su utilización debe decidirse individualmente. 
En todos los casos hay que conseguir el abandono del tabaco, de lo contrario la enfermedad seguirá un curso acelerado y además la producción de carboxihemoglobina disminuirá la disponibilidad de oxihemoglobina. Añadido al riesgo de incendio que supone la proximidad del oxígeno a un fuego hace que se considere que el no abandono del tabaco sea razón suficiente para el cese del tratamiento. Se ha estimado que aproximadamente el $20 \%$ de los pacientes en tratamiento con OCD continua fumando (9).

La OCD debe aplicarse al menos 15 h/día ya que los efectos en la supervivencia se logran a partir de ese nivel y no con una utilización más corta. Deben realizarse controles periódicos, cada 6 meses, en los que se insista en la necesidad del cumplimiento horario, ya que si no se hace así, el mero control periódico no consigue mejorar el cumplimiento horario. En estos controles es muy útil la realización de una Pulsioximetría que confirme la necesidad del tratamiento, si se observa una $\mathrm{SpO}_{2} 90 \%-92 \%$.

De todas forma la falta de una coincidencia exacta entre las cifras de la $\mathrm{SaO}_{2}$ obtenidos por medio de gasometría arterial y Pulsioximetría $\left(\mathrm{SpO}_{2}\right)$ o bien la no correspondencia exacta de la cifra de $\mathrm{PO}_{2}$ con la Pulsioximetría hacen que la utilización de esta sea motivo de discusión. Los pulsioximetrías actuales tienen una variabilidad de $\pm 1 \%-2 \%$ con respecto a la $\mathrm{SaO}_{2}$ de la gasometría arterial, a nivel de $\mathrm{SaO}_{2} 90 \%$. Así se observa que pacientes con la misma $\mathrm{SpO}_{2}$, tienen $\mathrm{pO}_{2}$ diferentes dentro unos limites. Hay una opinión mayoritaria de que una $\mathrm{SpO}_{2} \geq 92 \%$ significa casi con seguridad una $\mathrm{PO}_{2} \geq 60$ y que una $\mathrm{SpO}_{2}<92 \%$ significa que muy probablemente la $\mathrm{pO}_{2}$ es $<60 \%$ (sensibilidad 97\%-100\%; especificidad 86\%-100\%). Probablemente es aconsejable que con Sp90\%-92\% se realice una gasometría arterial ya que en ese rango hay casos con $\mathrm{pO}_{2}$ claramente superior a 60 y otros que apenas alcanzan 55, como se desprende de la lectura detenida del articulo de Feu y cols. (15-18).

Una $\mathrm{pO}_{2} \geq 60$ o una $\mathrm{SpO}_{2} \geq 92 \%$ son criterios para suspender la OCD ya que el paciente no la necesita. Hay que ser prudente cuando un paciente presenta coyunturalmente una $\mathrm{PO}_{2}>60$ en el curso de una historia de IRC ya que puede tratarse de un hecho aislado. En este caso si se suspende la OCD debe seguirse estrechamente al paciente (19).

Por otro lado la indicación inicial de oxigenoterapia suele realizarse en el curso de una reagudización, no en situación basal y eso lleva en muchos casos al mantenimiento a largo plazo de un tratamiento que puede no ser necesario.

En paciente sin hipoxemia basal pero con desaturaciones nocturnas no se han demostrado beneficios de la oxigenoterapia, ni en supervivencia ni en el desarrollo de hipertensión arterial pulmonar. Quizá en pacientes con IRC y OCD que presentan desaturación nocturna debiera aumentarse el flujo de oxígeno durante el sueño.

¿Qué nivel de desaturación nocturna, o durante cuando tiempo? O bien, ¿qué nivel de corrección de la desaturación nocturna, son necesarios para obtener un beneficio con los aumentos de flujo nocturno de oxigenoterapia? Son preguntas que de momento no tienen respuesta.

Eso sí, la presencia de desaturaciones periódicas durante la noche puede hacer pensar en la existencia de un proceso con alta prevalencia, la Apnea de sueño, donde un tratamiento con CPAP nocturna sí produciría claros beneficios.

Tampoco es clara la indicación de oxigenoterapia en pacientes con hipoxemia exclusivamente al hacer ejercicio físico. Aunque algunos trabajos muestran mejoría en el consu- mo de oxígeno máximo y disminución de la hiperinsuflación al suministrar oxigenoterapia otros estudios no han confirmado estos resultados ni una mejor evolución de la enfermedad.

Un grupo de pacientes con hipoxemia en reposo y que presentan agravamiento durante el esfuerzo pueden mejorar su capacidad de ejercicio con oxigenoterapia al disminuir las necesidades ventilatorias por un aumento de $\mathrm{FiO}_{2}$, por una mejor oxigenación de la musculatura, y en definitiva al disminuir la disnea. Sin embargo permanecen inciertas las indicaciones de oxigenoterapia durante el esfuerzo en este tipo de pacientes $(20,21)$. Es claro que la OCD no es el tratamiento de la disnea (22), pero es justo reconocer que hay pacientes en los que mejora la disnea al suministrar oxigenoterapia y en los que se encuentran razones fisiológicas y también un efecto placebo $(23,24)$.

Otra situación estimulante es la oxigenoterapia en el momento de la realización de ejercicios de rehabilitación respiratoria. Hay individuos que pueden beneficiarse, pero como grupo no se ha demostrado que los pacientes que la reciben tengan una mejor evolución que los que no lo hacen $(25,26)$.

Como fuentes suministradoras de oxígeno se recomiendan los concentradores (27) porque permiten mayor movilidad del paciente en el domicilio que las bombonas a presión y mayor independencia del suministrador. Los depósitos de oxígeno líquido permiten el llenado de pequeñas mochilas portátiles muy indicadas para pacientes buenos cumplidores, que realizan vida muy activa con muchas horas fuera de su domicilio y desaturación con el ejercicio. También en pacientes muy severos que precisan oxigenoterapia $24 \mathrm{~h} /$ día si pretenden salir del domicilio. En el País Vasco el 64\% de los tratamientos se realizan con concentradores, $29 \%$ con bombonas y $7 \%$ con oxígeno líquido, lo que nos sitúa lejos de la mayoría de los paises europeos donde la utilización de concentradores es masiva, pero en mejor situación que otras zonas, como la del grupo de Córdoba que se presenta en este número en el que la bombona constituye el $89 \%$ de los tratamientos.

\section{¿QUÉ SE ESPERA DE LA OCD?}

Fisiopatológicamente la oxigenoterapia evita la hipoxia y con ella evita o minimiza la vasoconstricción hipóxica pulmonar, el remodelado vascular, la hipertensión arterial pulmonar y la poliglobulia, lo que probablemente tiene mucho que ver con que este tratamiento es el único, junto con el abandono del tabaco, que consigue aumentar la supervivencia y mejorar la evolución en pacientes EPOC.

Aunque no existe una evidencia incuestionable, si hay estudios númerosos que indican que los pacientes mejoran la tolerancia al esfuerzo, la calidad de sueño y algunos funciones neuropsíquicos, entre ellos la memoria, así como una mejoría general en la calidad de vida, y no lo contrario, ya que es tentador pensar falsamente que la OCD puede suponer una esclavitud que empeora la calidad de vida (20).

En resumen, si indicamos bien la OCD, a los pacientes que realmente lo necesitan, éstos cumplen bien el tratamiento farmacológico y no fuman y utilizan el oxígeno al menos 15 h/día, conseguiremos que nuestros pacientes vivan más y mejor. Si además evitamos que pacientes que no necesitan oxigenoterapia, porque en situación basal tienen una $\mathrm{PO}_{2}>60$, no se vean sometidos a las incomodidades de este tratamiento, estaremos consiguiendo una correcta utilización de la OCD. 


\section{Bibliografía}

1. Levin BH, Bigelow DB, Hamstra RD, et al. The role of long term continuos oxygen administration in patients with chronic airway obstruction with hypoxemia. Ann Intern Med 1957; 66: 639.

2. Stark RD, Finnegan P, Bishop JN. Daily requirement of oxygen to reverse pulmonary hypertensión in patients with chronic broncitis. $\mathrm{Br}$ Med Jour 1972; 3: 147-157.

3. Nocturnal Oxygen Therapy Trial Group. Continous or nocturnal oxygen therapy in hypoxemic chronic obstrictive lung disease: a clinical trial. Ann Intern Med 1980; 93: 391-8.

4. Krop HD; Block JK, Cohen E. Neuropsichologic effects of continuous oxygen therapy in the aged. Chest 1977; 72: 737-43.

5. Report of the Medical Research Council Working Party. Long term domiciliary oxygen therapy in chronic hypoxic cor pulmonale complicating chronic bronchitis and enphysema. Lancet 1981; 1: 681-6.

6. Sánchez Agudo L, Cornudella R, Estopa R, Molinos L, Servera E. Normativa SEPAR para la indicación y empleo de la oxigenoterapia continua domiciliaria (OCD). Arch Bronconeumol 1998; 34: 87-94.

7. Escarrabill J, Estopa R, Huguet M, Riera J, Manresa F. Oxigenoterapia continua domiciliaria. Estudio de 344 pacientes. Arch Bronconeumol 1987; 23: 164-68.

8. Jurado B, Muñoz E, Davina S, Del Pino R, Ortiz A, Sans M. Oxigenoterapia domiciliaria. Estudio de utilización en un área sanitaria. Validación de la Pulsioximetría. An Med Interna 1998; 15: 358-62.

9. Granados A, Escarrabill J, Borras JM, Sánchez V, Jovell AJ. Utilización apropiada y efectividad: la oxigenoterapia crónica en Cataluña. Med Clin (Barc) 1996; 106: 251-3.

10. Sobradillo V, Miravitlles M, Gabriel R, Jiménez Ruiz C, Villasante C, Masa JF. Geographic variations in prevalence and underdiagnosis of COPD: result of the IBERPOC multicentre epidemiological study. Chest 2000; 118: 981-9.

11. Jaén JJ, de Castro C, Gontán MJ, López de Castro F. Prevalencia y factores de riesgo de EPOC en fumadores y exfumadores. Arch Bronconeumol 2003; 39: 554-8

12. SEPAR. Impacto social económico de la EPOC. SEPAR-Zambon Madrid 1995

13. Man SFP, McAlister FA, Anthonisen NR, Sin DD. Contemporary management of chronic obstructive pulmonary disease: Clinical applications. JAMA 2003; 290: 2313-6.

14. Pauwels RA, Buist AS, Calverley PM, Jenkins CR, Hurd SS. Global strategy for the diagnosis management and prevention of chronic obstructive pulmonary disease NHLBI/WHO. Global Initiative for Chronic Obstructive Lung Disease (GOLD) workshop summary. Am J Respir Crit Care Med 2001; 163: 1256-76.

15. Feu Collado MN, Jurado Gámez B, Rubio Sánchez J, Pascual Martínez N, Escribano Dueñas A, García Gil F, Muñoz Cabrera L. Rentabilidad de la pulsioximetría para el control de la oxigenoterapia domiciliaria. An Med Interna (Madrid) 2004; 21: 56-61.

16. Roberts CM, Bugler JR, Melchor R, Hetzel MR, Spiro SG. Value of pulse oximetry in screening for long-term oxygen therapy requirement. Eur Respir J 1993; 6: 559-62.

17. Kelly AM, McAlpine R, Kyle E. How accurate are pulse oximeters in patients with acute exacerbations of chronic obstructive airways disease? Respir Med 2001; 95: 336-40.

18. Roberts CM; Franklin J, O'Neill A, Roberts RLL, et al. Screening patients in general practice with COPD for long term oxygen requirement using pulse oxymetry. Respir Med 1998; 92: 1265-68.

19. Tarpy ST, Celli BR. Long term oxygen therapy. N Engl J Med 1995; 333: 710-14.

20. De Lucas P, Buendía MJ, Rodriguez JR. Oxigenoterapia: estado actua y controversias. Arch Bronconeumol 2002; 38 (Supl. 6): 31-6.

21. Wadell K, Henrikson-Larse K, Lundgren R. Physical training with and without oxygen in patients with chronic obstructive pulmonary disease and exercise-induced hypoxemia. J Rehabil Med 2001; 33: 200-5.

22. Shee CD. Palliation in chronic respiratory disease. Palliat Med 1995; 9 ; 3-12.

23. Waterhouse JC, Howard P. Breathlessness and portable oxygen in chronic obstructive airways disease. Thorax 1983; 38: 302-6.

24. Garrod R, Paul EA, Wedzicha JA. Supplemental oxygen during pulmonary rehabilitation in patiens with COPD with exercise hypoxemia. Thorax 2000; 55: 539-43.

25. Rooyakers JM, Dekhuijzen PNR; Van Herwarden CLA, Folgerin HTM Training with supplemental oxygen in patients with COPD and hypoxemia at peak exercise. Eur Respir J 1997; 10: 1278-84.

26. McDonald CF, Blyth CM, Lazarus MD, et al. Exertional oxygen of limited benefit in patients with chronic obstructive pulmonary disease and wild hipoxemia. Am J Respir Crit Care Med 1995; 152: 1616-9.

27. Escarrabill J. Oxigenoterapia domiciliaria: ¿a quién, cómo, cuándo, dónde y quién la controla? Arch Bronconeumol 1996; 32: 1-3. 